\title{
Los MOOC como herramientas de fortalecimiento en el aprendizaje de los Estudiantes de la carrera de Diseño Gráfico en la Facultad de Comunicación Social de la Universidad Guayaquil The MOOC as tools of strengthening in the learning of the Students of the Career of Graphic Design in the Faculty of Social Communication of the University Guayaquil
}

\author{
Jussen Paul Facuy Delgado 1,2,*, Ronny Enrique Santana Estrella ${ }^{2,3, \dagger}$, y Mirey Magdalena Cruz Ordoñez ${ }^{2, \ddagger}$ \\ ${ }^{1}$ Universidad Agraria del Ecuador. \\ ${ }^{2}$ Universidad de Guayaquil, Ecuador. \\ ${ }^{3}$ Escuela Superior Politécnica del Litoral, Ecuador. \\ \{jussen.facuy,ronny.santana.ec\}@gmail.com, mireyco@hotmail.com
}

Fecha de recepción: 8 de agosto de 2016 — Fecha de aceptación: 15 de agosto de 2016

\begin{abstract}
Resumen-El crecimiento acelerado que han tenido los cursos masivos en línea y abiertos MOOC Massive Open Online Course, ha modificado la manera de adquirir nuevos conocimientos, a través de múltiples plataformas de aprendizaje en línea, actualmente las universidades tienen la oportunidad de ampliar las posibilidades de otorgar una educación diferente a la tradicional, debido a la problemática que aqueja a nuestra sociedad, al existir limitaciones en el aprendizaje por múltiples factores que son desencadenados producto de la marginación y la pobreza, en muchos de los casos conlleva a una deserción educativa, por esta razón el deseo de implementar una nueva metodología de enseñanza aprendizaje que permita a los estudiantes el acceso a los medios de comunicación electrónicos como el internet y diferentes plataformas online disminuyendo de esta manera la brecha digital, diversificando con ello las opciones de formación y especialización. En este trabajo se documentan, los beneficios de los MOOC y por otro lado, se estudian aspectos que permiten a los estudiantes aprender desde cualquier lugar y en cualquier momento. En los nuevos escenarios educativos, los cursos universitarios no solo deben satisfacer las necesidades sociales-educativas que demanda un mundo globalizado, sino que las universidades deben desarrollar cursos que contemplen aspectos de internacionalización, donde se promueva la movilidad virtual de estudiantes y profesores, así como el reconocimiento internacional.
\end{abstract}

Palabras Clave-MOOC, E-Learning, Aprendizaje, Educación Superior.

Abstract-The accelerated growth of Massive Open Online Course online courses has changed the way to acquire new knowledge, through multiple online learning platforms, universities now have the opportunity to expand the possibilities of granting A different education to the traditional one, due to the problematic that afflicts our society, to exist limitations in the learning by multiple factors that are unleashed product of the marginalization and the poverty, In many cases leads to an educational desertion, for this reason the desire to implement a new methodology of teaching learning that allows students access to electronic media such as the internet and different online platforms thus decreasing the gap Digital, thus diversifying the options of training and specialization. This paper documents the benefits of MOOCs and, on the other hand, studies aspects that allow students to learn from anywhere and at any time. In the new educational scenarios, university courses should not only meet the social-educational needs demanded by a globalized world, but universities must develop courses that include aspects of internationalization, where virtual mobility of students and teachers is promoted, as well as The international recognition.

Keywords-MOOC, E-Learning, Learning, Higher Education.

\section{INTRODUCCIÓN}

$\mathbf{E}$ n los últimos años se han presentado diversos cambios tecnológicos que han modificado el medio como se relacionan los individuos en la sociedad. La nueva tendencia de cursos masivos abiertos en línea (MOOC) permite brindar capacitación abierta y en línea, innovando la educación superior. En las Universidades esto representa; un reto tecnológico y pedagógico, la incorporación de los MOOC en su oferta educativa, la interacción del alumno con el docente, el cambio

\footnotetext{
*Ingeniero en Computación e Informática, Magister en Finanzas y Proyectos Corporativos

${ }^{\dagger}$ Licenciado en Diseño Web y Aplicaciones Multimedia, Magister en Sistemas de Información Gerencial

${ }^{\dagger}$ Licenciada en Ciencias de la Educación Especialización Informática, Magíster en Informática Educativa
}

de pensamiento y práctica, así como la internacionalización que lleva de la mano la apertura de las diferentes culturas e idiosincrasias (Thirunarayanan M.O. et al., 2011).

Una de las estrategias competitivas que se implementó en la Carrera de Diseño Gráfico de la Facultad de Comunicación Social, es la incorporación en su metodología de enseñanza aprendizaje de los cursos MOOC, extendiéndose como un valor agregado a la producción, distribución y almacenaje de recursos educativos, creando ambientes de aprendizaje virtual, utilizando el internet como modo de comunicación, las redes sociales y plataformas educativas como medios de distribución de materiales educativos.

Este modelo educativo se fundamenta en el conectivismo desarrollado por Siemens, que lo denomina como la integración de los principios explorados por el caos, la red, la 
complejidad y teorías de auto organización, menciona que el aprendizaje y el conocimiento se basan en la diversidad de opiniones, que es un proceso de conectar nodos especializados o fuentes de información y la habilidad para ver conexiones entre campos, ideas y conceptos que son herramientas claves.

Muchos estudiantes tienen problemas en el aprendizaje en las diferentes asignaturas, una de las causas es que no hay atención individualizada debido a la heterogeneidad del grupo, factores de tiempo, habilidades y destrezas no desarrolladas, metodología tradicional aplicada por los docentes, debido que la Carrera de Diseño Gráfico está inmersa en las tendencias tecnológicas, necesita trabajar con el paradigma conectivista que está relacionado con los MOOC.

Por medio de los MOOC permitirá un aprendizaje que facilita la diseminación de la actividad de los participantes mediante el uso de una o varias plataformas, debido a que es un entorno gratuito y abierto, permite el apoyo masivo a la comunidad estudiantil por medio de la Diversidad de herramientas de comunicación, uso de redes sociales haciendo énfasis en el proceso de aprendizaje más que en la evaluación y acreditación.

\section{Objetivo}

Comparar y analizar el desarrollo de habilidades educativas aplicado a los estudiantes de Diseño Gráfico a través de las plataformas MOOC.

\section{Objetivos Específicos}

1. Identificar el nivel de habilidades técnicas en la pre prueba aplicando módulos virtuales en programas de educación superior de Diseño Gráfico.

2. Analizar el nivel en la post prueba aplicado módulos virtuales en programas de educación superior de Diseño Gráfico a través del MOOC.

3. Contribuir con el aprendizaje a través del acceso al Massive Online Open Courses (o Cursos online masivos y abiertos)

\section{DESARRollo}

\section{Características de los MOOC}

Existen básicamente dos tipos de cursos MOOC con base en su estructura y su metodología los xMOOC y los cMOOC. Pero hay ciertas diferencias entre los cursos del tipo cMoox y los de tipo xMooc: mientras los cursos cMoox (la c significa "conectivista") permiten una gran interacción entre sus participantes gracias a diversos recursos tales como compartir documentos en línea, escribir juntos en un blog o enviar / recibir FEEDS RSS para fortalecer su experiencia de aprendizaje, la colaboración entre los estudiantes de los cursos xMOOC (la x viene de las plataformas Edx, Coursera, Miriadax, entre otras) es un tanto más rígida debido a su estructura focalizada en una única página web que gestiona las acciones de todos los participantes del curso (Valles Alvarez et al., 2015).

\section{Plataformas para alojar MOOC}

La corriente de abrir los contenidos al mundo la conocemos como Open Course Ware. De tal manera que se empezaron a crear plataformas para alojar la gran cantidad de cursos creados, la idea de compartir los conocimientos, dio lugar a la plataforma tecnológica para alojar cursos MOOC, luego siguieron plataformas como: Aprendo, Coursera, Lore, Canavas Network, Open Class, edX, UniMOOC, UNX.

Tal principio de colocar en una plataforma los MOOC para una distribución masiva de materiales educativos se convirtieron en un cambio de modelo educativo del tradicional a un ambiente de aprendizaje virtual (Valles Alvarez et al., 2015).

El gran auge que ha mostrado en el sector educativo la incorporación de los MOOC y el desencadenamiento de liberar los materiales educativos para colocarlos en una plataforma y que sean de libre acceso, permitió la incorporación masiva de Universidades que ofrecen cursos MOOC como una alternativa para una educación de calidad, como es el caso de la Carrera de Diseño Gráfico de la Facultad de Comunicación Social el proceso de enseñanza-aprendizaje se ve envuelto por la gama inmensa de avances tecnológicos, principalmente en la forma de comunicación entre el docente y el estudiante, de tal manera que la interacción entre estos dos elementos facilita el intercambio de información, que a su vez crean redes interconectadas de conocimiento, lo que originó dar paso a la siguiente frase; un mismo estudiante puede obtener y generar conocimiento para compartirlo con otro estudiante. Este cambio revolucionario como método alterno de adquirir conocimiento en línea, lleva a un surgimiento masivo de Universidades que optan por ofrecer MOOC y concentrarlos en una plataforma.

\section{La competitividad y la Universidad}

Si bien las Universidades mantenían una competencia local por acaparar al mayor número de estudiantes y ofrecer mejores servicios, éstas pasaron de ser unos competidores locales a unos competidores nacionales o internacionales; la incorporación de las nuevas tecnologías de información y comunicación, producción y distribución de materiales con acceso abierto y libre, son factores que propician la incursión en un mercado educativo global. Si la competencia la asociamos a las Universidades, esto dependerá de la oferta educativa que a nivel global puedan ofrecer, de tal manera que, sus servicios educativos requieren la diversificación de opciones de enseñanza-aprendizaje para ampliar la cobertura a bajo costo, y la ventaja competitiva que tendrán dependerá en gran medida de la transformación de los recursos humanos, tecnológicos y materiales educativos con los que cuenta (Valles Alvarez et al., 2015).

\section{Desarrollo competitivo}

Las Universidades desarrollarán un ambiente competitivo global cuando sus actividades académicas están alineadas al uso de las TIC y la producción de recurso educativos, para ofrecer a los estudiantes diferentes opciones de aprendizaje. 
La internacionalización de la Universidad se ve reflejada por los cambios tecnológicos acelerados y por la necesidad de la sociedad de adquirir conocimientos, la globalización es un referente de la presencia internacional por los diferentes sectores que ejercen una competencia en ámbitos como lo económico, político, cultural y educativo. La producción y distribución de materiales educativos desde el interior de la Universidad crea ambientes de aprendizaje muy atractivos que la colocarán en una posición privilegiada para ofrecer a la sociedad, alumnos con conocimientos acorde a sus necesidades personales y a las demandas laborales mundiales.

\section{Internacionalización y movilidad digital}

La internacionalización en el sector educativo implica una serie de consideraciones que se deben de tomar en cuenta por parte de las Universidades, dentro de las que se encuentran, la economía, las creencias y costumbres, la globalización, la interculturalidad, la idiosincrasia de los pueblos, sociedades y naciones, las cuales van a conformar una comunicación internacional. Han existido numerosas definiciones de internacionalización, con aplicación en diferentes sectores tanto en lo económico, político, social y educativo. La internacionalización en las instituciones es definida como el proceso de integrar una internacionalización, interculturalidad o dimensión global dentro de un propósito, funciones o entrega de una educación post-secundaria.

La movilidad estudiantil establece el traslado de estudiantes de una universidad local a una universidad foránea, siendo ésta última en el mismo territorio nacional o en el extranjero. Las Universidades con un sistema de movilidad estudiantil, cuentan con sus propios lineamientos para ofrecer a los alumnos la posibilidad de trasladarse físicamente a una Universidad que cumpla con los objetivos profesionales y académicos.

Esta necesidad de movilidad virtual surge de acuerdo a las necesidades para adquirir nuevos conocimientos y para satisfacer las demandas educativas, teniendo la opción de escoger entre un número considerable de Universidades que cuentan con esta modalidad. El internet es uno de los principales elementos para que haya una movilidad virtual, las redes sociales juegan un papel importante en la comunicación entre el estudiante y la Universidad en la cual se inscribirá. Los elementos a considerar en una movilidad virtual son de carácter tecnológico, básicamente contar con las herramientas adecuadas para la comunicación, como una computadora personal y acceso a internet, las habilidades personales que muestren en el uso de las TIC los alumnos y maestros y el portal educativo que la Universidad tenga disponible para albergar a los huéspedes digitales. Otro aspecto importante es la modificación de los planes y programas para alumnos virtuales, la accesibilidad a bibliotecas virtuales y la disponibilidad de los recursos tecnológicos con los que se cuente, el cambio significativo y quizá radical es centrar los recursos tecnológicos educativos en los estudiantes.

Las redes sociales son una herramienta que se creó con el objetivo de compartir información, las cuales muy pronto se convirtieron en el primer medio de comunicación en internet. Existen diferentes entornos sociales con los cuales se puede compartir información de manera inmediata y con un sin número de participantes. Según Prensky, los nativos digitales piensan, aprenden y viven de manera diferente de quienes les enseñan, que son migrantes digitales (Valles Alvarez et al., 2015).

\section{Producción de MOOC}

Existen diferentes vías para la elaboración de un curso MOOC, esto dependerá de las necesidades que tengan los alumnos y de las herramientas tecnológicas con las que cuente la Universidad. Existen requisitos mínimos para su construcción, en el curso "Introducción a los MOOC". Esta estructura se compone principalmente de dos bloques, el primero se relaciona con elementos, evaluación y certificación de un MOOC y presenta los siguientes elementos: estructura de un MOOC, el rol docente en un MOOC, la evaluación en los MOOC y la certificación. El segundo bloque se relaciona con el diseño y creación de un mini MOOC y presenta los siguientes elementos: determinar el tema y tipo de destinatario, determinar contenidos, elementos necesarios para componer un contenido didáctico como una unidad mini MOOC, determinar herramientas para realizar la interacción con los participantes, planificar temporalización, determinar actividades y sistemas de interacción con el alumnado, determinar sistemas de presentación y difusión, selección de espacios para albergar los recursos de un mini MOOC, y selección y manejo de herramientas para convertir un material didáctico en un mini (Valles Alvarez et al., 2015).

Un aspecto que hay que considerar es el tema de la evaluación, para este tipo de cursos se toman como métricas de evaluación: la participación en foros de discusión, entrega de tareas en tiempo y forma, responder a cuestionarios relacionados con el tema, la evaluación por pares y las autoevaluaciones.

Para la producción es importante contar con recursos tecnológicos adecuados como son computadoras, cámaras de video, software para procesamiento de video y de audio, el considerar estos recursos garantizan en gran medida una buena producción de un curso MOOC. Aún y cuando los cursos MOOC son de libre acceso y se puede acceder a ellos en cualquier momento, es importante considerar los tiempos que deban destinarse para el inicio y el término del mismo, una calendarización de actividades, definir los momentos de entrega de actividades, la asesoría grupal y momento de practicar la evaluación como parte del seguimiento del curso MOOC (Valles Alvarez et al., 2015).

El personal de enseñanza o de tutoría, es el responsable de realizar las siguientes actividades: el diseño instruccional del curso MOOC y en ocasiones es el que inicia y brinda la asesorías o tutorías a los alumnos inscritos, administra el aprendizaje en los foros de discusión, brinda seguimiento a las actividades de aprendizaje, también son los responsables de las evaluaciones al final del curso MOOC.

\section{Recursos Educativos Abiertos}

La UNESCO sostiene que el acceso universal a la educación de gran calidad es esencial para la construcción de la paz, el desarrollo sostenible de la sociedad y la economía y el diálogo intercultural. Los recursos educativos de libre acceso 
proporcionan una oportunidad estratégica para mejorar la calidad de la educación y para facilitar el diálogo sobre políticas, el intercambio de conocimientos y el aumento de capacidades (UNESCO, 2002). "Los Recursos Educativos Abiertos (REA) son recursos destinados para la enseñanza, el aprendizaje y la investigación que residen en el dominio público o que han sido liberados bajo un esquema de licenciamiento que protege la propiedad intelectual y permite su uso de forma pública y gratuita o permite la generación de obras derivadas por otros. Los Recursos Educativos Abiertos se identifican como cursos completos, materiales de cursos, módulos, libros, video, exámenes, software y cualquier otra herramienta, materiales o técnicas empleadas para dar soporte al acceso de conocimiento (UNESCO, 2002).

\section{Beneficios de MOOC}

Los MOOC son cursos elaborados principalmente por profesores e investigadores con un diseño instruccional de la propia institución educativa con el objetivo de ofertarlos a los estudiantes de una forma masiva a través de la red de internet (Rodriguez, 2016).

- El objetivo principal es romper las barreras de un salón de clases y llegar al mayor número de estudiantes posibles.

- Los MOOC son una herramienta muy importante como estrategia de crecimiento y presencia a nivel mundial, brinda los beneficios de contar con un número considerable de alumnos, da a conocer a todos los internautas los servicios educativos con los que cuentan la Carrera de Diseño Gráfico de la Facultad de Comunicación Social, tiene la visión de crear ambientes de aprendizaje abiertos.

- Al ser una herramienta nueva viene a ser un complemento de la educación tradicional, con el uso de las herramientas tecnológicas se crean registros de los estudiantes y permiten conocer cuáles son sus necesidades particulares de aprendizaje y con ello brinda la posibilidad de crear cursos acorde a los intereses y necesidades de los estudiantes.

- Tiene la posibilidad de contar con cursos de calidad con materiales educativos elaborados por los docentes expertos en las materias.

\section{MATERIAL Y MÉTODOS}

La metodología MOOC, al tener ausente la figura del tutor online, hace difícil establecer un seguimiento o nota tal y cómo se ha ido haciendo hasta ahora. De momento, gracias a este tipo de herramientas, podemos ir compensando esta figura, tan necesaria inicialmente para la creación del material, pero menos importante en el proceso de autoaprendizaje del alumno. Aprendizaje autónomo pero en comunidad (SCOPEO, 2013).

Las entrevistas tienen una duración máxima de 3 minutos. Las preguntas realizadas fueron las siguientes:

- ¿Cuál es su experiencia con los MOOC?

- Cómo ve el futuro de esta tendencia, ¿irá a más o es algo temporal?

- ¿MOOC implica más negocio o MOOC implica más aprendizaje?
El Focus Group tuvo una duración aproximada de una hora y cuarto, procurando que el proceso permitiera la participación de todos los expertos entrevistados. El procedimiento consistió en la realización de rondas de preguntas, otorgando la palabra de forma ordenada, al objeto de obtener valoración de cada uno de los participantes en el focus group. Por su duración, no se realizó ninguna parada.

Los temas a tratar durante el Focus Group son los siguientes:

- Primeramente se ha delimitado: Qué es un MOOC y qué no es un MOOC?

- ¿Por qué los MOOC ahora? ¿Qué es lo que ha hecho que surja esta nueva oleada de cursos masivos online?

- Cuál es la manera en que las entidades, ya sean universidades o empresas, puedan afrontar este cambio, si es que lo van a afrontar... ¿Qué pasos hay que seguir?, ¿qué es lo que NO se debería hacer? Y ¿qué es lo que se está haciendo mal?

- Cómo puede afectar esta nueva metodología de enseñanza a la educación en general y más en particular, a la enseñanza universitaria. ¿Qué es lo que nos queda por ver? O ¿lo hemos visto todo?

- Se está hablando mucho y escribiendo sobre que los MOOC son un negocio, ¿Cómo se entiende esta afirmación desde un punto de vista más corporativo?

- Esto tiene un fin muy significativo e innegable que es la educación y el aprendizaje, ¿los MOOC mejoran el aprendizaje de los alumnos? Es decir, tienen un valor añadido a la enseñanza presencial o a otro tipo de cursos online "tradicionales".

- Tipo de investigación: Experimental

- Diseño: Cuasi experimental (Grupo pre experimental con pre y post prueba).

- Población: Estudio a realizarse a los estudiantes de Diseño Gráfico de la Universidad de Guayaquil 60 personas

- Finalidad de Estudio: La investigación tiene por finalidad demostrar que los MOOC generará un desarrollo de habilidades y mejorará el conocimiento a los estudiantes.

- Instrumentos: Pre Prueba, Post Prueba, Encuestas o cuestionario (Opinión sobre el modulo).

- Modelo matemático: Datos Estadísticos (T-Student para comparación de grupos, diferencia de medias, porcentuales y frecuencias) probabilístico.

\section{CONCLUSIONES}

Las aulas tradicionales que comúnmente conocemos con pizarras y tizas liquidas, hoy en día ya no son los recursos educativos suficientes, debido a que los estudiantes y docentes debemos responder a nuevas necesidades y estrategias didácticas, ofreciendo una enseñanza mixta, presencial y virtual. Los MOOC que ofrecen las plataformas Coursera, Udacity, EdX, entre otros brindan cursos basados en el modelo de realización de actividades y difusión del conocimiento de acuerdo a las necesidades particulares de cada individuo. La plataforma ha contado con una prueba piloto de tres cursos masivos y online, tales como Dispositivos Móviles, Diseño Web y Computación Aplicada al Diseño (PSD, .AI, FL, ETC) El aprendizaje es el resultado de la comprensión de los contenidos, la práctica de 
tareas automatizadas y la participación colaborativa, basados en la teoría conectivista, o cMOOC. La importancia de integrar a los docentes y estudiantes a través de una plataforma MOOC, dará como resultados que todos los contenidos académicos de la carrera Diseño Gráfico sean ofrecidos bajo esta modalidad.

\section{REFERENCIAS BIBLIOGRÁFICAS}

Rodriguez, D. (2016). MOOC - emaze.

SCOPEO (2013). MOOC: estado de la situación actual, posibilidades, retos y futuro.

Thirunarayanan M.O., Elizabeth C. Ferris, Stanley P. (Pat) Pressey, and Wellington Andrees (2011). Transactional Distance Theory: Should It Be Revised To Exclude StudentStudent Interactions? 8(6):3-4.

UNESCO (2002). Recursos educativos abiertos - Organización de las Naciones Unidas para la Educación, la Ciencia y la Cultura.

Valles Alvarez, M., Fernando, S., and Arturo Amaya Amaya, M. (2015). Beneficios de los MOOC en Educación Superior. Memorias del Encuentro Internacional de Educación a Distancia, 4(4). 